\title{
How can we detect hydrogen bond local cooperativity in liquid water: a simulation study
}

Imre Bakó, ${ }^{\mathrm{a} *}$ Anikó Lábas, ${ }^{\mathrm{b}}$ Kersti Hermansson ${ }^{\mathrm{c}}$, Ákos Bencsura, ${ }^{\mathrm{a}}$ Julianna Oláh ${ }^{\mathrm{b} *}$

a. Institute of Organic Chemistry Research Centre for Natural Sciences, Hungarian Academy of Sciences, Magyar tudosók körútja 2 519, P.O. Box 286 Budapest, Hungary

b. Department of Inorganic and Analytical Chemistry, Budapest University of Technology and Economics, H-1111 Szent Gellért tér 4., Budapest, Hungary

c. Department of Chemisty, Ångström Laboratory Box 538. S-75121 Uppsala Sweden

\begin{abstract}
The significant cooperative effect between water molecules substantially affects the properties of liquid water. The cooperativity of hydrogen bonds means that the hydrogen bond strength is influenced by the neighboring water molecules. Another descriptor related to cooperativity is degree correlation (or static correlation) describing the probability of hydrogen-bonded molecule pairs participating in additional hydrogenbonds. Herein we analyze the latter one in liquid water at various temperatures and densities in a series of molecular dynamics simulations with the help of knowledge from network science. We investigated how the applied hydrogen bond criteria (energetic or geometric) influence the obtained results, and showed that the energetic criterion is much more rigorous and reliable, therefore should be used for similar studies. We found that the structure of the subsystems of water molecules with 3 and 4 hydrogen-bonds is distinctly different at low temperature, 3-hydrogen-bonded water molecules form branched chain structures at all temperature. Deconvolution of the descriptors of the mixing pattern of water molecules according to their donor and acceptor numbers showed that species with complementary hydrogen bonding properties are likely to correlate and form H-bonds with each other, while species with similar H-bond pattern tend to avoid each other. Pearson's coefficient (global descriptor of the local cooperativity) of the studied networks suggests that at normal density the H-bonded network in liquid water can be described by an uncorrelated network.
\end{abstract}




\section{Introduction}

It is generally understood that the unique physical, chemical and structural properties of liquid water originate from the existence of a complex and dynamic threedimensional hydrogen bonded (H-bonded) network. Liquid water shows many anomalies in its thermodynamical and dynamical properties, which become even more pronounced in the supercooled region, such as the density decrease or the increase of diffusivity upon isothermal compression. A comprehensive overview of these anomalies has been compiled by Chaplin. ${ }^{1}$ Four different scenarios (1) the stability limit ${ }^{2}$ the liquid-liquid critical point ${ }^{3}(3)$ the singularity free ${ }^{4-7}$ and the (4) critical point free ${ }^{8}$ scenarios have been put forward to explain the origin of these anomalies. Furthermore, various speculations suggest that the cooperativity among $\mathrm{H}$-bonds has an important role in determining the anomalous properties of water. ${ }^{9-14}$ Indeed, Stokely et al. ${ }^{15}$ has showed that the strength of the cooperative component of the H-bond and the strength of the directional component of the H-bond determine which of the four scenarios mentioned above describes water properly at a given state.

The cooperativity of water molecules has been in the focus of intensive theoretical research and recently broadband rotational spectroscopy has also been successfully applied to obtain experimental evidence for this phenomenon. ${ }^{16}$ The concept of H-bond cooperativity, has originally been suggested by Frank and Wen, ${ }^{17}$ and means that the local H-bond strength is influenced by the neighboring water molecules. As a consequence, the interaction energy of two water molecules also depends on their interaction with a third, or several additional water molecules, making the three-body terms essential in the evaluation of the overall interaction energy. Further manifestations of the cooperativity of $\mathrm{H}$-bonds include changes of electronic properties such as dipole moment or changes in the $\mathrm{O}-\mathrm{H}$ vibrational frequencies. ${ }^{18}$ These phenomena have been extensively investigated by quantum mechanical calculations and ab initio molecular dynamics simulations.

Other descriptors related to cooperativity might also be defined. The correlation between $\mathrm{H}$-bonds describes the probability of $\mathrm{H}$-bonded molecule pairs participating in additional H-bonds. Degree correlation (static correlations) in real networks indicates that 
the links are not randomly wired and the probabilities of the existence of a bond among nodes with different degrees deviate from their expectation value. The correlation between H-bonds could be investigated by assessing the mixing properties of the system and comparing the degree-degree correlation function of the H-bond network to the random distribution. This quantity can be used to measure the local cooperativity among the species. Luzar showed that in the range of statistical uncertainties the number of $\mathrm{H}$ bonds around a specified molecule is essentially uncorrelated with the number of $\mathrm{H}$ bonds around a bonded nearest neighbor. ${ }^{19}$ She also studied single H-bond dynamics by the reactive flux formalism and found no dynamical cooperativity between different $\mathrm{H}$ bonds in water. ${ }^{20}$ Errington et. al. ${ }^{21,22}$ found that formation of more tetrahedral regions in liquid water is cooperative. Raiteri et. al. ${ }^{23}$ showed that cooperative processes (dynamical cooperativity) between neighboring $\mathrm{H}$-bonds are significant on short time scales and they become more pronounced at low temperatures.

Recently substantial insight has been given into the underlying connectivity properties of various networks, including biological, sociological and engineering systems as well. ${ }^{24-28}$ Generally speaking, a network can be represented by a graph built up from vertices and links. In the simplest description the network has a binary nature, where the edges (bonds) between the nodes are either present or not. Many real systems can be better described by a weighted graph $^{29}$ in which each bond carries a numerical value measuring the strength of the connection. In the case of liquid water, vertices are the water molecules and the links are the H-bonds between them.

Graph theoretical algorithms have been recently gaining larger role in the investigation of the structural properties of hydrogen-bonded liquids. Bakó and coworkers applied a spectral graph theory to investigate H-bonding network topology in liquid water, ${ }^{30,31}$ methanol, ${ }^{30}$ formamide, ${ }^{32}$ and water-formamide ${ }^{33}$ and water-methanol ${ }^{34}$ mixtures and around proteins ${ }^{35}$. Clark et al applied other essential topological indices (geodesic distribution - the shortest H-bond pathway between molecular vertices) to investigate the $\mathrm{H}$-bonded network in water, methanol, ethanol, and their binary mixtures adsorbed in microporous hydrophobic zeolite and $\alpha$-quartz|water interfaces. ${ }^{36}$ They have also developed the ChemNetworks software, which is a general code to convert chemical systems to chemical network formalism. ${ }^{37}$ Choi and Cho used spectral graph analysis to 
study the mechanism of ion aggregation in high salt concentration solutions and found that the ion clusters formed in $\mathrm{NaCl}$ solution are graph-theoretically and morphologically different from the ion network structures in KSCN solution. ${ }^{38}$

In this paper we investigate how temperature and pressure influences the static correlation between H-bonds in liquid water. In order to do so, we carried out molecular dynamics simulations in various temperatures and densities on liquid water, and transformed the Cartesian coordinates of the snapshots into a network of H-bonds. Finally, the obtained H-bond networks were analyzed using the tools of network science. The transformation requires a definition for $\mathrm{H}$ bonds. In the literature various definitions of H-bond exist, thus we decided to check how using the geometric and energetic criteria for H-bonds would influence the most profound properties of the graphs representing the H-bond network. 


\section{Computational details}

Molecular dynamics simulations: Molecular dynamics (MD) simulations in NVT ensemble were performed using the DL_POLY 2.20 program. ${ }^{39}$ Each system consisted of 2048 molecules in a cubic box corresponding to three different densities: 1.0, 1.12 and $1.24 \mathrm{~g} / \mathrm{cm}^{3}$. The simulations were carried out at all densities at three different temperatures: at 250,300, and $350 \mathrm{~K}$, respectively. Periodic boundary conditions were employed and the Ewald summation was used to handle the long-range Coulomb interactions. The short-range interactions were cut-off at $15 \AA$.

The MD trajectories were generated using the $\mathrm{SPC} / \mathrm{E}^{40}$ intermolecular potential model for water with a time step of fs. The boxes were equilibrated for $1 \mathrm{~ns}$ and data collected for $5 \mathrm{~ns}$ at $300 \mathrm{~K}$ and $350 \mathrm{~K}$ and $10 \mathrm{~ns}$ at $250 \mathrm{~K}$ using the Verlet leap-frog algorithm under control of the Nose-Hoover thermostat with a weak coupling $\tau=0.5 \mathrm{ps}$. Longer simulation time was used at $250 \mathrm{~K}$ due to the increased life of the H-bonds in order to obtain improved sampling. Rigid-body constraints were maintained using the quaternion algorithms.

MD trajectories were analyzed using two, frequently applied ${ }^{41}$, different H-bond definitions. In our work two molecules were considered $\mathrm{H}$-bonded if (1) the $\mathrm{O} \cdots \mathrm{H}$ distance was smaller than $2.5 \AA$ and the interaction energy was smaller than $-3.0 \mathrm{kcal} / \mathrm{mol}$ (energetic definition) (2) the $\mathrm{O} \bullet \bullet \mathrm{H}$ distance was shorter than $2.5 \AA$, and the $\mathrm{H}-\mathrm{O} \bullet \bullet \mathrm{O}$ angles less than $30^{\circ}$ (geometric definition) (3) the $\mathrm{O} \bullet \bullet \cdot \mathrm{H}$ distance was shorter than $2.5 \AA$, and the $\mathrm{O} \bullet \bullet \mathrm{O}$ distance was shorter than $3.35 \AA$ (additional geometric definition). Furthermore, we investigated the sensitivity of our conclusions and of the H-bonded properties of the system to the applied geometrical and energetic parameters $(\mathrm{H}-\mathrm{O} \ldots \mathrm{O}$ angle between $20^{\circ}-40^{\circ}$, interaction energy from $-1.5 \mathrm{kcal} / \mathrm{mol}$ to $\left.-3.5 \mathrm{kcal} / \mathrm{mol}\right)$. The statistically significant difference between the results obtained from the two datasets were assessed by Student's t-test and F-test.

H-bond network analysis: We carried out a statistical analysis of the graphs representing the $\mathrm{H}$-bond network of liquid water that were obtained from the MD simulations. Every 2 ps a snapshot has been taken from the MD trajectory at $300 \mathrm{~K}, 350$ $\mathrm{K}$ and $250 \mathrm{~K}$, in order to avoid short-term correlation between the configurations, then they were converted to graphs of the H-bond network (links: H-bonds, nodes: water 
molecules) resulting in 2500 and 5000 graphs in total, respectively at $300 / 350 \mathrm{~K}$ and at $250 \mathrm{~K}$. For each graph we determined the average H-bond number $\left(\mathrm{n}_{\mathrm{HB}}\right)$, the fraction of water molecules with a given number of $\mathrm{H}$-bonds $\left(\mathrm{f}_{\mathrm{k}}\right)$, where index k refers to the number of H-bonded neighbors of a given water molecule, and from this the H-bond number distribution $\left(\mathrm{f}_{\mathrm{hb}}(\mathrm{i})\right)$. Additionally we analyzed the distribution of donor and acceptor $\mathrm{H}$ bond number $(\mathrm{n}(\mathrm{I}, \mathrm{J})$, where $\mathrm{I}$ and $\mathrm{J}$ define the number of donor and acceptor sites of a given water molecule). ${ }^{42}$

Networks are frequently represented by weighted graphs. ${ }^{34}$ In H-bonded networks the interaction energy of the $\mathrm{H}$-bond $\left(\mathrm{E}_{\mathrm{HB}}\right)$ arises as the most suitable quantity to be used as weight. For this reason the interaction energy between each bonded pair was assessed individually to yield $\mathrm{E}_{\mathrm{HB}}$ using the SPC/E force field, and was used to create the weighted graphs, to calculate the average $\mathrm{H}$-bond strength $\left.\left(\left\langle\mathrm{E}_{\mathrm{HB}}\right)\right\rangle\right)$, the $\mathrm{H}$-bond strength distribution $\left(\mathrm{P}\left(\mathrm{E}_{\mathrm{HB}}\right)\right)$ and the strength of a node $\left(\mathrm{E}_{\text {total }}\right)$ (total interaction energy: the sum of the interaction energies of a water molecule with its $\mathrm{H}$-bonded neighbors calculated from pair-wise interaction energies). The strength of a node integrates the information about its connectivity and the weights of its links. The more negative the overall interaction energy of the central molecule is, the stronger the node is. The percolation properties of the systems were also studied 


\section{Results and discussion}

We carried out a detailed analysis of the H-bonding properties of the nine investigated systems. The number of average H-bonded neighbor number $\left(\mathrm{n}_{\mathrm{hb}}\right)$ decreases as a function of temperature being in good agreement with other studies ${ }^{43-48}$ (see Table 1.). Interestingly, in contrast to previous assumptions the H-bond definition affects this quantity considerably, as the more restrictive energetic definition yields on average 0.5 unit smaller $n_{h b}$ values than the geometric definition. Further difference between the results is that using the energetic definition the fraction of water molecules with three $\mathrm{H}$ bonds $\left(f_{3}\right)$ is the most substantial, while the geometric definition predicts water molecules with four H-bonds $\left(f_{4}\right)$ to be the most frequent. Prompted by these discrepancies we thoroughly investigated the results obtained by the two definitions (see the Supplementary material for the detailed discussion). In short, we found that the none of the geometric definitions fulfills properly the necessary requirements (directional and always attractive in character) for a H-bond (see Table S2 in the Suppl. Mat.). Therefore, from now on we use the energetic definition of the H-bond throughout our paper for analyzing our simulation data. Here we want to remark that we carried out all analysis using both the energetic and the geometric definition in order to assess the effect of used H-bond definition on all our conclusion and as we showed in the supplementary material, that the main conclusions do not depend on the applied definitions

Table 1. Characteristic values of H-bonded networks in liquid water at different temperatures at $1.0 \mathrm{~g} / \mathrm{cm}^{3}$ density $\left(\mathrm{n}_{\mathrm{hb}}\right.$ : average number of H-bonds, $\mathrm{f}_{\mathrm{k}}$ fraction of water molecules with a given number $(\mathrm{k})$ of $\mathrm{H}$-bonds)

\begin{tabular}{|l|l|r|r|r|r|r|}
\hline & \multicolumn{1}{|c|}{ HB definition } & $\mathrm{f}_{2}$ & $\mathrm{f}_{3}$ & $\mathrm{f}_{4}$ & $\mathrm{f}_{5}$ & $\mathrm{n}_{\mathrm{HB}}$ \\
\hline $250 \mathrm{~K}$ & $2.5 \AA, 30^{\circ}$ & 0.066 & 0.302 & 0.563 & 0.062 & 3.61 \\
\hline & $2.5 \AA,-3 \mathrm{kcal} / \mathrm{mol}$ & 0.171 & 0.417 & 0.366 & 0.019 & 3.18 \\
\hline $300 \mathrm{~K}$ & $2.5 \AA, 30^{\circ}$ & 0.129 & 0.371 & 0.423 & 0.056 & 3.37 \\
\hline & $2.5 \AA,-3 \mathrm{kcal} / \mathrm{mol}$ & 0.250 & 0.419 & 0.252 & 0.017 & 2.91 \\
\hline $350 \mathrm{~K}$ & $2.5 \AA, 30^{\circ}$ & 0.192 & 0.394 & 0.323 & 0.046 & 3.14 \\
\hline & $2.5 \AA,-3 \mathrm{kcal} / \mathrm{mol}$ & 0.310 & 0.389 & 0.180 & 0.015 & 2.68 \\
\hline
\end{tabular}


The characteristic values for describing the H-bond network of liquid water at various temperatures and densities are presented in Table 2. The average number of $\mathrm{H}$ bonds slightly increases as a function of density at a 300 and $350 \mathrm{~K}$, but decreases at $250 \mathrm{~K}$. At all temperatures and densities the fraction of water molecules with three $\mathrm{H}$ bonds is the most substantial, it is about $40 \%$. At all temperatures the fraction of water molecules with one and two H-bonds decrease with increasing density while the fraction of water molecules with four and five $\mathrm{H}$-bonds increase, in line with our expectations.

An interesting aspect of the H-bond network structure is the donor and acceptor number of the various nodes, the probabilities of the most frequent joint donor/acceptor numbers are also shown in Table 2. E.g. n(1,2) refers to the fraction of those water molecules which donate $1 \mathrm{H}$-bond and accept $2 \mathrm{H}$-bonds. We do not show the $\mathrm{n}(2,2)$ values as it is almost equal to $\mathrm{f} 4$. The fraction of water molecules with $5 \mathrm{H}$-bonds increases significantly as a function of density, and it is almost equal with $\mathrm{n}(2,3)$. Water molecules with $3 \mathrm{H}$-bonds can be divided into two significantly different populations, namely $\mathrm{n}(2,1)$ and $\mathrm{n}(1,2)$, the first one being larger in all cases. The probability of both types of nodes decreases slightly as a function of temperature. Our finding agrees well with data obtained by Markovich et al at $300 \mathrm{~K} .{ }^{42}$

Table 2 Characteristic values of H-bonded networks in liquid water at different temperatures and densities $\left(\mathrm{n}_{\mathrm{hb}}\right.$ :average H-bond number, $\mathrm{f}_{\mathrm{k}}$ fraction of water molecules with a given number $(\mathrm{k})$ of $\mathrm{H}$-bonds, $\mathrm{n}(\mathrm{I}, \mathrm{J})$ : fraction of water molecule with I donor and $\mathrm{J}$ acceptor site)

\begin{tabular}{|c|c|c|c|c|c|c|c|c|c|}
\hline & & $f_{2}$ & $f_{3}$ & $f_{4}$ & $f_{5}$ & $\mathrm{n}_{\mathrm{hb}}$ & $\mathrm{n}(1,2)$ & $\mathrm{n}(2,1)$ & $n(2,3)$ \\
\hline \multirow[t]{3}{*}{$250 \mathrm{~K}$} & 1.00 & 0.171 & 0.417 & 0.366 & 0.019 & 3.18 & 0.178 & 0.244 & 0.017 \\
\hline & 1.12 & 0.180 & 0.420 & 0.343 & 0.026 & 3.16 & 0.178 & 0.241 & 0.025 \\
\hline & 1.24 & 0.187 & 0.420 & 0.324 & 0.034 & 3.14 & 0.178 & 0.241 & 0.030 \\
\hline \multirow[t]{3}{*}{$300 \mathrm{~K}$} & 1.00 & 0.250 & 0.419 & 0.252 & 0.017 & 2.91 & 0.176 & 0.241 & 0.016 \\
\hline & 1.12 & 0.247 & 0.415 & 0.251 & 0.023 & 2.93 & 0.175 & 0.241 & 0.022 \\
\hline & 1.24 & 0.243 & 0.41 & 0.252 & 0.030 & 2.96 & 0.174 & 0.236 & 0.029 \\
\hline \multirow[t]{3}{*}{$350 \mathrm{~K}$} & 1.00 & 0.310 & 0.392 & 0.180 & 0.015 & 2.68 & 0.163 & 0.228 & 0.014 \\
\hline & 1.12 & 0.295 & 0.391 & 0.194 & 0.020 & 2.75 & 0.165 & 0.225 & 0.019 \\
\hline & 1.24 & 0.283 & 0.389 & 0.205 & 0.028 & 2.80 & 0.166 & 0.222 & 0.027 \\
\hline
\end{tabular}




\section{Percolation properties of water molecules with three and four $\mathrm{H}$-bonds.}

Several authors suggested that the water molecules with four H-bonds form small ramified patches whose density is lower than that of water (low density patches). We showed in our earlier studies ${ }^{31}$ that in the case of SPC/E water potential these molecules almost form a percolated network at $250 \mathrm{~K}$ and at $1.0 \mathrm{~g} / \mathrm{cm}^{3}$ indicated by the similar cluster size distribution of the low-density patches at $250 \mathrm{~K}$ as the cycle size distribution of the overall HB structure of water at ambient temperature. However, as the fraction of water molecules belonging to cyclic entities was found to be significantly smaller than the same quantity in bulk water.

Here, we extended this analysis for the subsystems including only 3 or $4-\mathrm{H}$ bonded molecules. Molecules were considered to be part of a given a connected entity if there was a minimum length path consisting of a series of $\mathrm{H}$-bonds through which one can reach all of the other water molecule of the entity. In Fig. 1 we show the cluster size distribution of water molecule with 3 and $4 \mathrm{H}$-bonds at $1.24 \mathrm{~g} / \mathrm{cm} 3$, but similar distributions were obtained at smaller densities as well. It is known that in a percolated network the cluster size distribution has a significant bump at larger cluster sizes, indicating the appearance of very large clusters which we only observed in the case of 4 $\mathrm{H}$-bonded water molecules at $250 \mathrm{~K}$. The structure of the population of water molecules with $3 \mathrm{H}$-bonds is branched chain at all 3 temperatures.

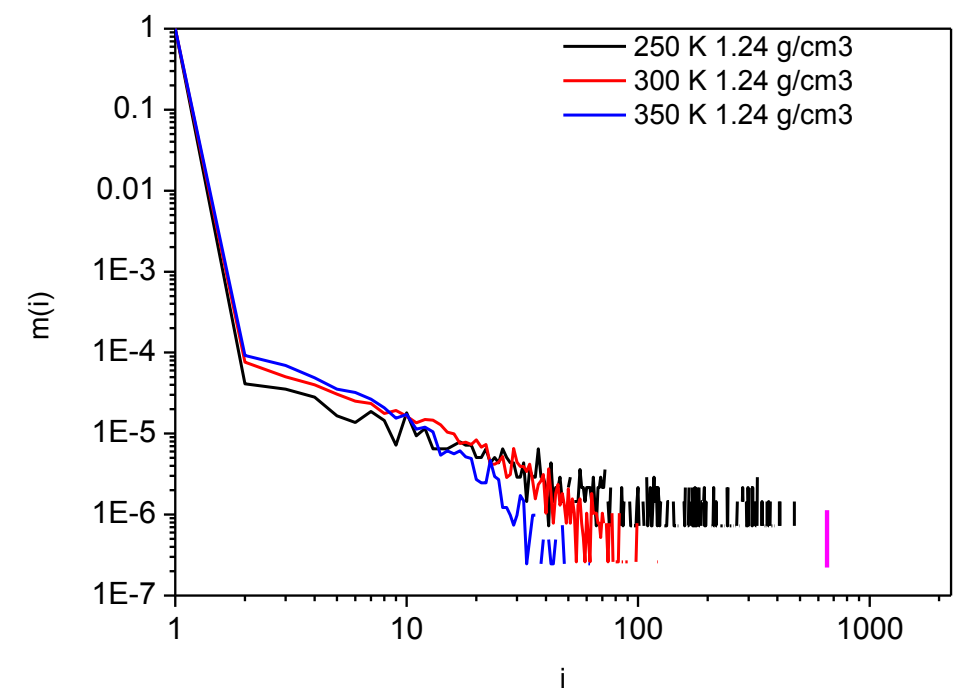

A. 


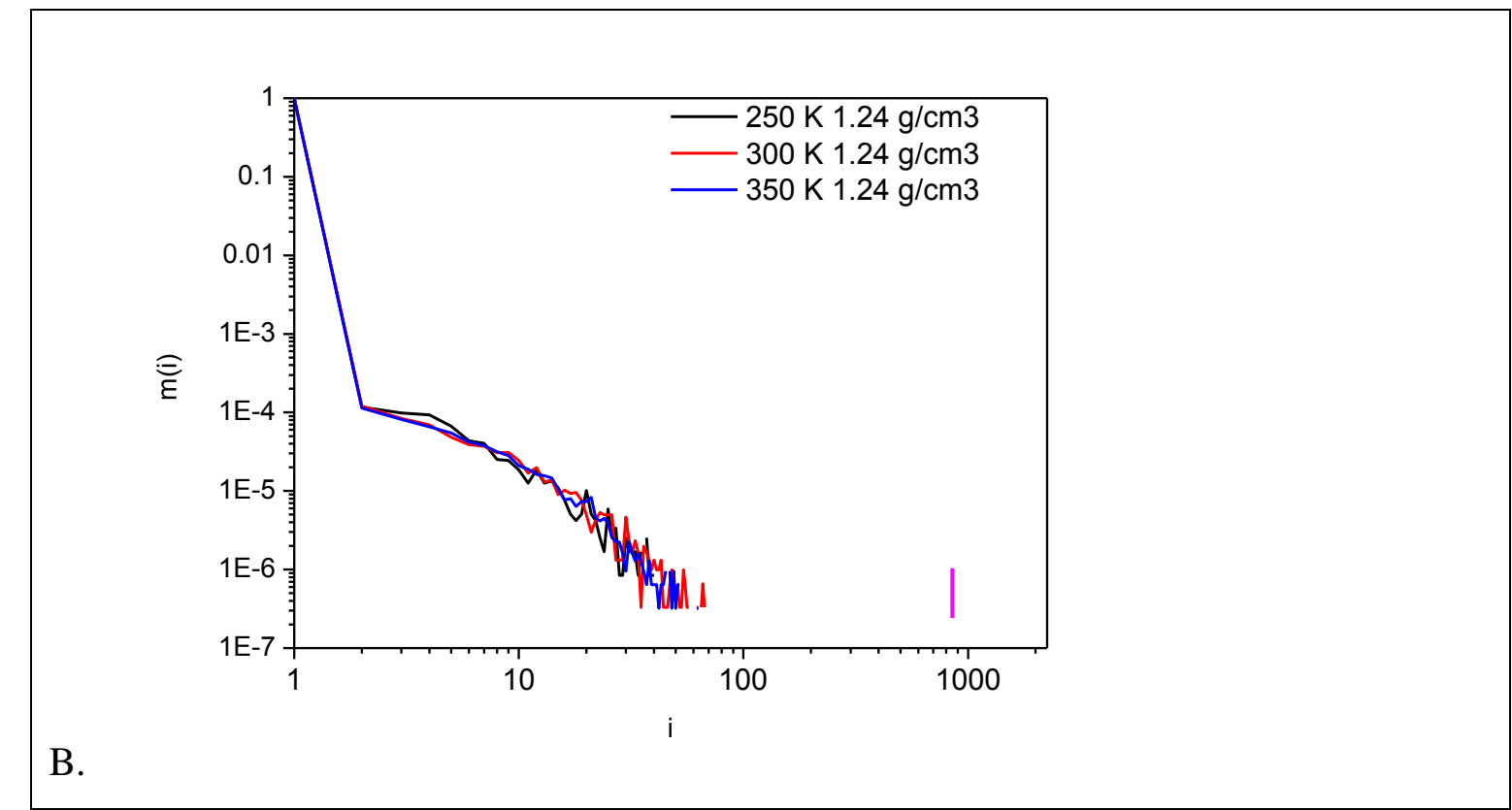

Fig 1. Cluster size distribution $\mathrm{m}(\mathrm{i})$ in liquid water at various temperatures for 4 H-bonded (A.) and $3 \mathrm{H}$-bonded (B.) water molecules at $1.24 \mathrm{~g} / \mathrm{cm}^{3}$. The purple vertical lines indicate the number of 4 and $3 \mathrm{H}$-bonded molecules in the system. 


\section{H-bond interaction energy as a function of the H-bonded environment.}

The average total interaction strength of water molecules $\left(\mathrm{E}_{\mathrm{total}}\left(\mathrm{n}_{\mathrm{HB}}\right)\right)$ as a function of $\mathrm{H}$ bonded neighbor number is shown in Fig. 2, and its distribution function in the Supplementary Material (Fig. S3). Each investigated system exhibits the same type of $\mathrm{E}_{\text {total }}\left(\mathrm{n}_{\mathrm{HB}}\right)$ dependence. The average interaction energy of water molecules forming a single $\mathrm{H}$-bond is about $-4.8 \mathrm{kcal} / \mathrm{mol}$ which is somewhat less favorable, due to the nonideal geometries visited in the MD trajectory, than the interaction energy of -6.57 $\mathrm{kcal} / \mathrm{mol}$ obtained at the optimum geometry using the SPC/E force field. ${ }^{36,49}$ The formation of the second, third and fourth $\mathrm{H}$-bonds leads to a very similar energy gain as the first, but the obtained function starts to deviate considerably from linearity at H-bond number 5 because of the steric requirements for a $\mathrm{H}$-bond. Presence of a fifth hydrogen bonding water molecule distorts the first solvation shell of the central molecule, which leads to the elongation of the original H-bond distances, thus to their weakening. The almost linear correlation between the total strength of the node (the sum of the interaction energies between the central molecule and its H-bonded neighbours) and the H-bond neighbor number (between one and four) implies that most $\mathrm{H}$-bonds are characterized by very similar interaction energies, thus each link has approximately the same weight regardless of the network topology. 


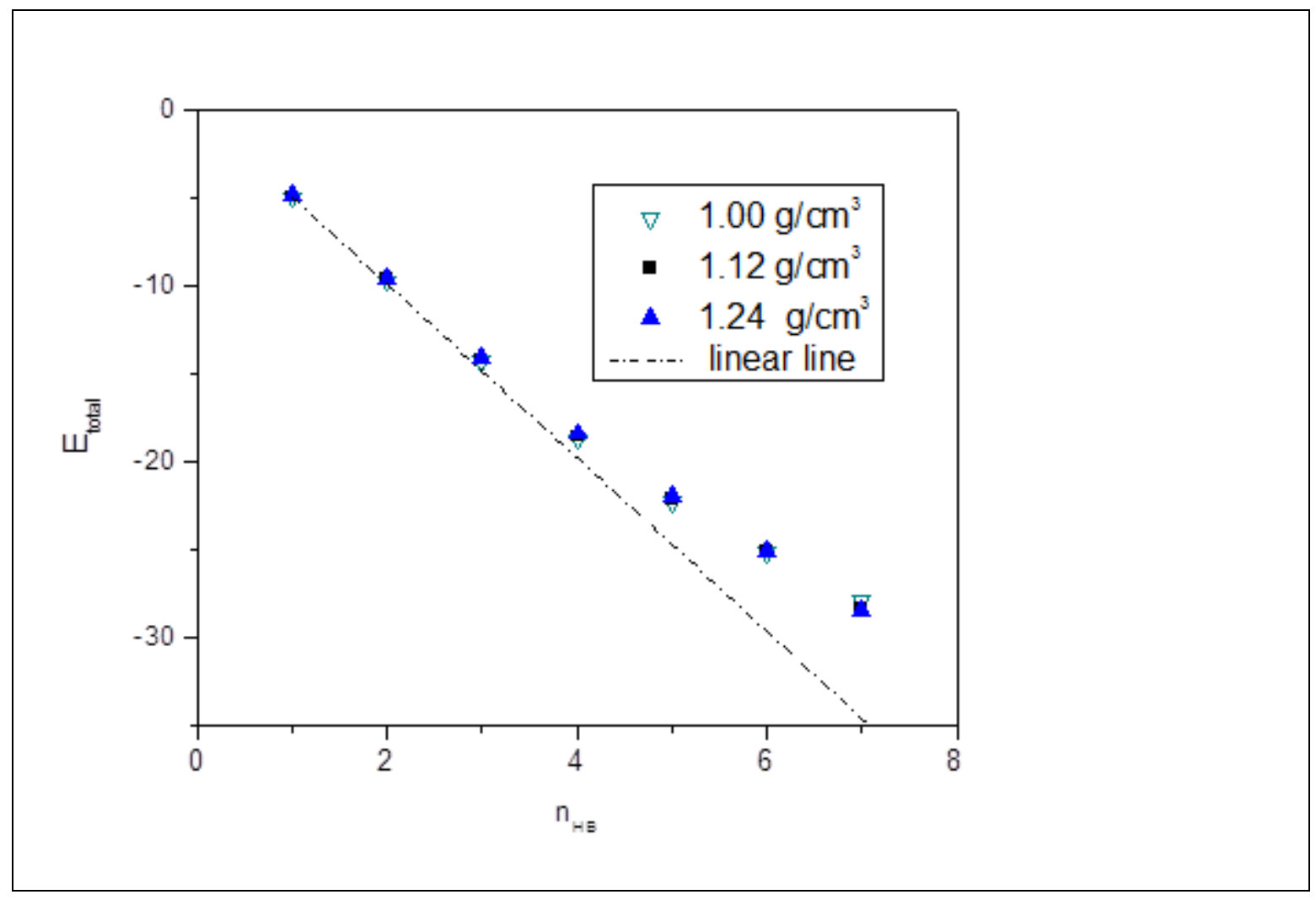

Fig. 2. The average total interaction energy (strength of node) of water molecules $\left(E_{\text {total }}\right.$ $\mathrm{kcal} / \mathrm{mol}$ ) as a function of $\mathrm{n}_{\mathrm{hb}}$, the number of $\mathrm{H}$-bonded neighbors at $300 \mathrm{~K}$.

We decomposed the interaction energy data as a function of the donor/acceptor number $(n(I, J))$ of the water molecules (Table 3.). The interaction energy between two water molecules significantly depends on their H-bonded environment. The calculated differences are statistically significant in all cases (as showed by Student's t-test and the F-test) and show a continuous increase as a function of temperature and density. The strongest interactions at all temperatures and densities are observed between species with lowest number of H-bonds: between species with $\mathrm{n}(\mathrm{I}, \mathrm{J})$ 1,1 and 1,1; 1,1 and 2,1; 2,1 and 2,2. It is somewhat unexpected to detect the reasonably large (about $0.4 \mathrm{kcal} / \mathrm{mol}$ ) interaction energy difference between molecular pairs with 1,2-1,2 and 2,1-2,1 donor/acceptor numbers in all investigated systems. Our results, presented in the Supplementary material (Table S4), revealed that this conclusion did not depend on the applied H-bond criteria, but is a general property of the SPC/E force field. 
This result is in contrast to the conclusions of quantum chemical calculations. Ohno et al. proposed a relationship between the H-bonded state of donor and acceptor molecule and the strength of the H-bond and found that the strongest H-bond and largest red shift of the $\mathrm{OH}$ stretching frequency occur in the 1.2-2,1 pair.$^{50}$ This conclusion was also drawn by Tao et al. ${ }^{51}$ The failure of the SPC/E model to properly describe the Hbonded interaction locally in liquid water, compared to the quantum chemical calculation is not a surprising results, as the SPC/E water model does not include polarization and charge transfer terms. It is very probable that this is one of the reasons, why the SPC/E model does not describe properly the phase diagram of liquid water ${ }^{52}$

Table 3. The average H-bond interaction energy between the different water species $(\mathrm{kcal} / \mathrm{mol}), \mathrm{n}(\mathrm{I}, \mathrm{J})$ : I: number of donor sites, J: number of acceptor sites. A1: acceptor molecule, D1: donor molecule

\begin{tabular}{|r|r|r|r|r|r|r|r|r|r|r|}
\hline $\mathrm{D} 1$ & $\mathrm{~A} 1$ & \multicolumn{3}{|c|}{$1.0 \mathrm{~g} / \mathrm{cm} 3$} & \multicolumn{3}{|c|}{$1.12 \mathrm{~g} / \mathrm{cm} 3$} & \multicolumn{3}{|c|}{$1.24 \mathrm{~g} / \mathrm{cm} 3$} \\
\hline $\mathrm{I}, \mathrm{J}$ & $\mathrm{I}, \mathrm{J}$ & $250 \mathrm{~K}$ & $300 \mathrm{~K}$ & $350 \mathrm{~K}$ & $250 \mathrm{~K}$ & $300 \mathrm{~K}$ & $350 \mathrm{~K}$ & $250 \mathrm{~K}$ & $300 \mathrm{~K}$ & $350 \mathrm{~K}$ \\
\hline $\mathbf{1 , 2}$ & $\mathbf{1 , 2}$ & -4.80 & -4.68 & -4.58 & -4.77 & -4.66 & -4.57 & -4.75 & -4.65 & -4.57 \\
\hline $\mathbf{1 , 2}$ & $\mathbf{2 , 1}$ & -4.85 & -4.73 & -4.63 & -4.83 & -4.71 & -4.62 & -4.80 & -4.69 & -4.60 \\
\hline $\mathbf{1 , 2}$ & $\mathbf{2 , 2}$ & -4.79 & -4.68 & -4.58 & -4.76 & -4.66 & -4.57 & -4.73 & -4.63 & -4.55 \\
\hline $\mathbf{1 , 2}$ & $\mathbf{2 , 3}$ & -4.66 & -4.55 & -4.47 & -4.64 & -4.54 & -4.47 & -4.61 & -4.53 & -4.45 \\
\hline $\mathbf{2 , 1}$ & $\mathbf{2 , 1}$ & -5.14 & -5.01 & -4.90 & -5.10 & -4.97 & -4.86 & -5.05 & -4.93 & -4.82 \\
\hline $\mathbf{2 , 1}$ & $\mathbf{2 , 2}$ & -4.89 & -4.78 & -4.68 & -4.86 & -4.75 & -4.65 & -4.82 & -4.72 & -4.63 \\
\hline $\mathbf{2 , 1}$ & $\mathbf{2 , 3}$ & -4.59 & -4.51 & -4.44 & -4.58 & -4.50 & -4.43 & -4.56 & -4.49 & -4.43 \\
\hline $\mathbf{2 , 2}$ & $\mathbf{2 , 2}$ & -4.79 & -4.68 & -4.58 & -4.75 & -4.65 & -4.56 & -4.72 & -4.62 & -4.53 \\
\hline $\mathbf{2 , 2}$ & $\mathbf{2 , 3}$ & -4.57 & -4.49 & -4.39 & -4.55 & -4.47 & -4.39 & -4.53 & -4.45 & -4.38 \\
\hline $\mathbf{1 , 1}$ & $\mathbf{1 , 1}$ & -5.20 & -5.05 & -4.92 & -5.17 & -5.02 & -4.90 & -5.12 & -4.99 & -4.87 \\
\hline $\mathbf{1 , 1}$ & $\mathbf{1 , 2}$ & -4.92 & -4.80 & -4.69 & -4.90 & -4.78 & -4.68 & -4.87 & -4.76 & -4.67 \\
\hline $\mathbf{1 , 1}$ & $\mathbf{2 , 1}$ & -5.17 & -5.03 & -4.91 & -5.13 & -4.99 & -4.87 & -5.09 & -4.95 & -4.84 \\
\hline $\mathbf{1 , 1}$ & $\mathbf{2 , 2}$ & -4.98 & -4.86 & -4.74 & -4.94 & -4.82 & -4.72 & -4.90 & -4.79 & -4.70 \\
\hline
\end{tabular}

\section{Investigation of cooperativity effect in $\mathrm{H}$-bonded network}

It is known, that many real networks are markedly different in their mixing patterns. Social networks such as Facebook and scientific co-authorships show strong assortative character, meaning that highly connected nodes are likely to form links with similarly highly connected nodes. In contrast, protein interaction networks and neural 
networks show a disassortative character meaning that high degree nodes tend to attach to low degree nodes.

One can determine the assortative character of the network, by introducing the conditional probability $\mathrm{P}^{\text {calc }}\left(\mathrm{k}, \mathrm{k}^{\prime}\right)$, where $\mathrm{k}$ is the number of $\mathrm{H}$-bonded neighbors, to define the probability that a bond from a molecule characterized with $\mathrm{k}$ H-bonded neighbor number points to another molecule characterized with k' H-bonded neighbor number. In an uncorrelated network, this conditional probability has the following form:

$$
P^{u n c}\left(k, k^{\prime}\right)=\frac{k f_{h b}(k) k^{\prime} f_{h b}\left(k^{\prime}\right)}{\sum_{k, k^{\prime}} k f_{h b}(k) k^{\prime} f_{h b}\left(k^{\prime}\right)}
$$

The static cooperation effect (excess conditional probability) can be characterized by the difference between the conditional probabilities $\mathrm{P}^{\text {calc }}(\mathrm{k}, \mathrm{k}$ ') calculated directly from the simulation or from the H-bond number distribution as calculated from Eq. 1.

The excess conditional probability has the following form.

$$
\Delta P^{\text {exc }}\left(k, k^{\top}\right)=P^{\text {calc }}\left(k, k^{\top}\right)-P^{u n c}\left(k, k^{\top}\right)
$$

It is known, that the direct evaluation of $\mathrm{P}^{\text {calc }}\left(\mathrm{k}, \mathrm{k}^{\prime}\right)$ distribution gives extremely noisy results for a real network and a H-bonded network of water. ${ }^{27}$ In Fig. 3. we compare the $\mathrm{P}^{\text {calc }}\left(\mathrm{k}, \mathrm{k}^{\prime}\right)$ values calculated directly from the simulation with the $\mathrm{P}^{\mathrm{unc}}\left(\mathrm{k}, \mathrm{k}^{\prime}\right)$ calculated from the $\mathrm{H}$-bond number distribution. It is evident that the statistical uncertainties of these quantities are too large, therefore the calculated deviations given in Table 4 are not satisfactorily well-defined. This conclusion for liquid water at $300 \mathrm{~K}$, and at $1.00 \mathrm{~g} / \mathrm{cm}^{3}$ density has already been showed by Luzar for a significantly smaller-sized system and using a shorter simulation time and without additional statistical analyses. ${ }^{19,20}$ We carefully analyzed our data statistically (applying Student's t-test and Ftest), so we can conclude that a significant positive excess conditional probability (extra correlation) exists between water molecules with $4 \mathrm{H}$-bonds at $250 \mathrm{~K}$ at every density. A positive extra correlation means that the probability of finding a link between the given nodes is higher than would be expected in a random graphs, thus at low temperatures 
water molecules with $4 \mathrm{H}$-bonds are very likely to form $\mathrm{H}$-bonds with other water molecules with $4 \mathrm{H}$-bonds. This certainly points into the same direction as the results of the cluster size analysis: which showed that if only the subsystem of water-molecules with 4-H-bonds is investigated very large, extended rings can be found (seen in Fig. 1)., in contrast to the subsystem of three-hydrogen-bonded water molecules. Furthermore, this finding also supports the idea of the presence of the low density patches formed by water molecules with four $\mathrm{H}$-bonds. This extra correlation between the water molecules with $3 \mathrm{H}$-bonds is negative, smaller and statistically not significant in several cases. These results are in good agreement with our earlier data, namely, that water molecule with $3 \mathrm{H}$-bonds do not form a percolated network at any temperature.

Table 4. Characteristic values of the excess $\Delta \mathrm{P}^{\mathrm{exc}}\left(\mathrm{k}, \mathrm{k}^{\prime}\right)$ function calculated from simulations at various temperatures and densities. Statistically significant data are shown in italics.

\begin{tabular}{|c|c|c|c|c|c|c|c|c|}
\hline \multirow[t]{4}{*}{$1.00 \mathrm{~g} / \mathrm{cm}^{3}$} & & $2 \ldots .3$ & $2 . .4$ & $3 . .3$ & $3 \ldots 4$ & $3 . . .5$ & $4 . .4$ & $4 . .5$ \\
\hline & $250 \mathrm{~K}$ & $3.1 E-04$ & $-9.6 E-04$ & $-4.7 \mathrm{E}-04$ & $-2.7 E-04$ & $3.9 E-04$ & $2.2 E-03$ & $-8.6 E-04$ \\
\hline & $300 \mathrm{~K}$ & $2.8 \mathrm{E}-04$ & $-4.7 E-04$ & $-8.4 E-04$ & $5.6 E-04$ & $-3.7 E-05$ & $3.5 \mathrm{E}-04$ & $-3.7 E-04$ \\
\hline & $350 \mathrm{~K}$ & $3.0 \mathrm{E}-04$ & $-4.3 E-04$ & $-3.0 \mathrm{E}-04$ & $1.7 \mathrm{E}-04$ & $-3.4 E-04$ & $1.2 \mathrm{E}-04$ & $2.2 E-04$ \\
\hline \multirow[t]{3}{*}{$1.12 \mathrm{~g} / \mathrm{cm}^{3}$} & $250 \mathrm{~K}$ & $4.0 E-04$ & $-8.8 E-04$ & $-3.6 \mathrm{E}-04$ & $-1.1 \mathrm{E}-04$ & 8.6E-05 & $1.7 E-03$ & $-5.6 E-04$ \\
\hline & $300 \mathrm{~K}$ & $3.7 E-04$ & $-7.6 E-04$ & $-2.2 \mathrm{E}-04$ & $2.6 \mathrm{E}-04$ & $-4.2 E-04$ & $6.7 E-04$ & $-2.3 \mathrm{E}-05$ \\
\hline & $350 \mathrm{~K}$ & $4.9 E-04$ & $-7.6 E-04$ & $-7.5 \mathrm{E}-05$ & $2.1 \mathrm{E}-04$ & $-6.2 E-04$ & $2.7 \mathrm{E}-04$ & $3.8 E-04$ \\
\hline \multirow[t]{3}{*}{$1.24 \mathrm{~g} / \mathrm{cm}^{3}$} & $250 \mathrm{~K}$ & $7.4 E-04$ & $-9.6 E-04$ & $2.8 \mathrm{E}-04$ & $-2.2 E-04$ & $-6.7 E-04$ & $1.6 E-03$ & $-1.8 \mathrm{E}-04$ \\
\hline & $300 \mathrm{~K}$ & $7.9 E-04$ & $-1.0 E-03$ & $4.7 \mathrm{E}-04$ & $-7.6 \mathrm{E}-05$ & $-1.0 E-03$ & $9.3 E-04$ & $2.9 E-04$ \\
\hline & $350 \mathrm{~K}$ & $9.0 E-04$ & $-1.2 E-03$ & $7.4 E-04$ & $-2.9 E-04$ & $-1.2 E-03$ & 8.0E-04 & 7.6E-04 \\
\hline
\end{tabular}




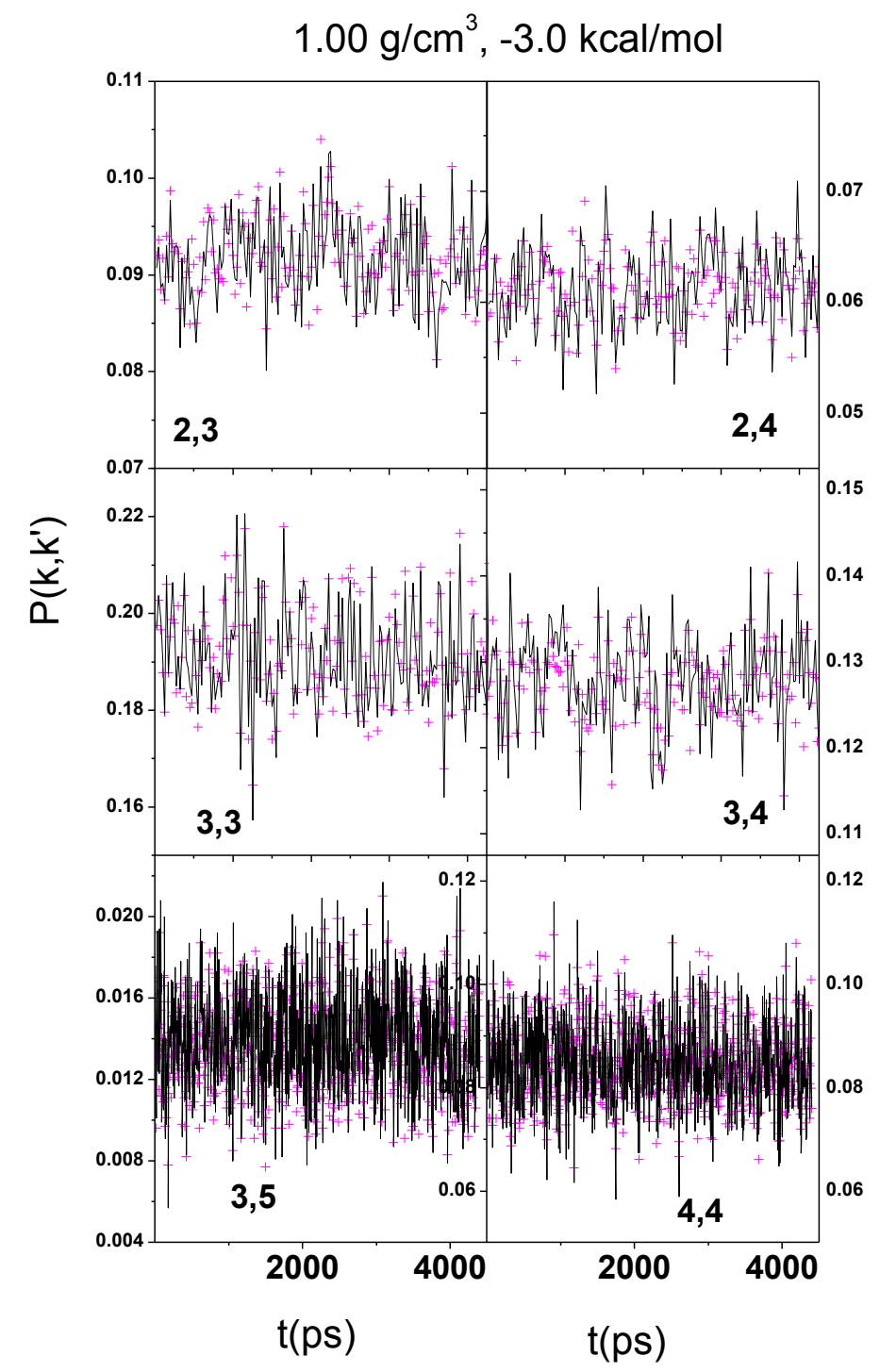

Fig. 3. $\mathrm{P}\left(\mathrm{k}, \mathrm{k}^{\prime}\right)$ functions for the system at $1.00 \mathrm{~g} / \mathrm{cm}^{3}$ density and at $\mathrm{T}=300 \mathrm{~K}$ calculated from the simulation $\left(\mathrm{P}^{\mathrm{calc}}\left(\mathrm{k}, \mathrm{k}^{\prime}\right)\right.$, black line) or directly from Eq. $1\left(\mathrm{P}^{\mathrm{unc}}(\mathrm{k}, \mathrm{k})\right.$, pink crosses).

In order to get more detailed information about the correlation between species with 3 and $3 \mathrm{H}$-bonds (3..3) and 3 and $4 \mathrm{H}$-bonds (3...4) we decomposed the $\mathrm{P}(\mathrm{k}, \mathrm{k}$ ') data according to donor and acceptor number ( $\mathrm{I}$ and $\mathrm{J}$ ) of the species participating in H-bond formation. This led to the following main pairs: $1,2 \ldots 1,2,1,2 \ldots 2,1,2,1 \ldots 2,1,1,2 \ldots 2,2$, $2,1 \ldots 2,2$. E.g. the pair 1,2 ..1,2 indicates the interaction between a species with $3 \mathrm{H}$ bonds participating once as a donor and twice as acceptor interacting with another water 
molecule with $3 \mathrm{H}$-bonds (1 donor and 2 acceptor sites). The calculated excess quantities are given in Table 5. A significant positive correlation exists between 1,2...2,1 and $2,1 \ldots 2,2$ species and negative correlation between $1,2 \ldots 1,2 ; 2,1 \ldots 2,1$ and $1,2 \ldots 2,2$ species. Positive correlation implies that species are likely to form hydrogen bonds (as it is found for species with complementary H-bonding pattern), while species with similar hydrogen bond pattern are likely to avoid each other (negative correlation). The very weak or statistically not significant excess correlation among the 3,3 and 3,4 species results from the cancellation of various correlations with different signs.

Table 5. Characteristic values of the excess $\mathrm{P}(\mathrm{I}, \mathrm{J}, \mathrm{I}$ 'J') function calculated from simulations at various temperatures and densities (all of the data are statistically significant., I,J: I:donor number, J: acceptor number)

\begin{tabular}{|r|r|l|l|l|l|l|}
\hline density & $\mathrm{T}(\mathrm{K})$ & $1,2 \ldots 1,2$ & $1,2 \ldots 2,1$ & $2,1 \ldots 2,1$ & $1,2 \ldots 2,2$ & $2,1 \ldots 2,2$ \\
\hline & 250 & $-3.43 \mathrm{E}-03$ & $1.06 \mathrm{E}-02$ & $-7.71 \mathrm{E}-03$ & $-1.29 \mathrm{E}-03$ & $4.39 \mathrm{E}-04$ \\
\hline $1.00 \mathrm{~g} / \mathrm{cm}^{3}$ & 300 & $-4.39 \mathrm{E}-03$ & $1.27 \mathrm{E}-02$ & $-9.20 \mathrm{E}-03$ & $-1.27 \mathrm{E}-03$ & $2.32 \mathrm{E}-03$ \\
\hline & 350 & $-4.64 \mathrm{E}-03$ & $1.32 \mathrm{E}-02$ & $-8.87 \mathrm{E}-03$ & $-1.41 \mathrm{E}-03$ & $1.96 \mathrm{E}-03$ \\
\hline & 250 & $-3.37 \mathrm{E}-03$ & $1.06 \mathrm{E}-02$ & $-7.65 \mathrm{E}-03$ & $-7.42 \mathrm{E}-04$ & $3.44 \mathrm{E}-04$ \\
\hline $1.12 \mathrm{~g} / \mathrm{cm}^{3}$ & 300 & $-4.11 \mathrm{E}-03$ & $1.24 \mathrm{E}-02$ & $-8.57 \mathrm{E}-03$ & $-9.33 \mathrm{E}-04$ & $1.54 \mathrm{E}-03$ \\
\hline & 350 & $-4.41 \mathrm{E}-03$ & $1.23 \mathrm{E}-02$ & $-8.09 \mathrm{E}-03$ & $-7.62 \mathrm{E}-04$ & $1.52 \mathrm{E}-03$ \\
\hline & 250 & $-3.29 \mathrm{E}-03$ & $1.05 \mathrm{E}-02$ & $-7.14 \mathrm{E}-03$ & $-4.50 \mathrm{E}-04$ & $2.01 \mathrm{E}-04$ \\
\hline $1.24 \mathrm{~g} / \mathrm{cm}^{3}$ & 300 & $-3.84 \mathrm{E}-03$ & $1.18 \mathrm{E}-02$ & $-7.48 \mathrm{E}-03$ & $-5.07 \mathrm{E}-04$ & $9.52 \mathrm{E}-04$ \\
\hline & 350 & $-4.11 \mathrm{E}-03$ & $1.17 \mathrm{E}-02$ & $-7.10 \mathrm{E}-03$ & $-6.65 \mathrm{E}-04$ & $9.71 \mathrm{E}-04$ \\
\hline
\end{tabular}

The problem caused by the statistical uncertainties of excess conditional probabilities calculated directly from the simulation could be overcome by defining the average nearest neighbor degree distribution ( $\mathrm{k}_{n n, i}$ eq. (3)) or the average weighted nearest neighbors degree ( $k_{n n, i}^{w}$, eq. (3)) of node $i$ in the following forms:

$k_{n n, i}=\frac{1}{k_{i}} \sum_{j} k_{j}$

and

$$
k_{n n, i}^{w}=\frac{1}{E_{t o t a l, i}} \sum_{j} E_{h b, i j} k_{j}
$$

where the summation goes over all $\mathrm{H}$-bonded neighbors and

$$
E_{\text {total,i }}=\sum_{j} E_{h b, i j}
$$


and $\mathrm{E}_{\mathrm{hb}, \mathrm{ij}}$ is the interaction energy between the $\mathrm{i}$-th and the $\mathrm{j}$-th water molecules. If there is no degree correlation, then $k_{n n}(k)$ is independent of $\mathrm{H}$-bond number $k$ and it is equal to $\left\langle n_{h b}{ }^{2}\right\rangle /\left\langle n_{h b}\right\rangle$. Correlated graphs are called assortative (disassortative) if $k_{n n}(k)$ is increasing (decreasing) as a function of $\mathrm{k}$. This correlation can be quantified by using the numerical slope of $k_{n n}(k)$, which is also called the Pearson correlation coefficient.

In Fig.4a we have depicted the dependence of the average nearest neighbor degree distribution on the H-bonded neighbor number. In most cases the Pearson correlation coefficient is around zero showing no statistically significant assortative or disassortative mixing character. However, at larger densities (especially at $1.24 \mathrm{~g} / \mathrm{cm}^{3}$ ) the coefficient becomes positive (around 0.02 at all temperatures) showing a slight cooperation. The conclusions are the same for both the weighted (see Fig. 4) and unweighted (not shown) graphs. 


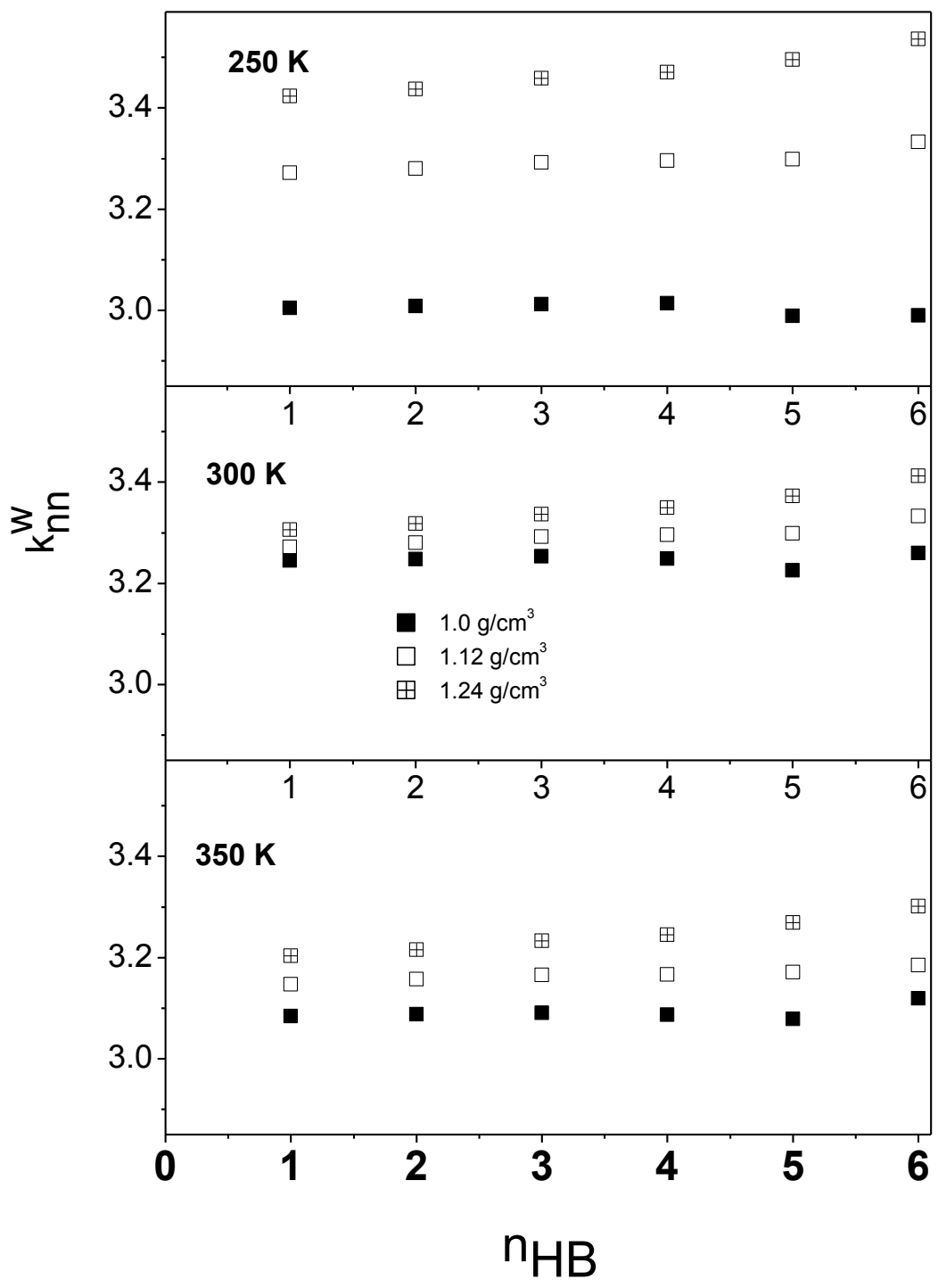

Fig. 4. Cooperation in the water network at different temperatures and densities. Data obtained from a weighted network. 


\section{Conclusions}

In the present paper we applied a method to the study the cooperative properties of the H-bond networks in liquid water which is widely used in network science for characterizing the mixing properties of real networks (social, technological and biological networks). We investigated how the applied H-bond criteria (energetic or geometric) influences the obtained results, and showed that the energetic criterion is much more rigorous and reliable, therefore its use is strongly recommended for the study of hydrogen bond networks. We found a significant change in the shape of the H-bond interaction energy distribution curve of water molecules incorporated in different H-bonding environments.

Analysis of the cluster size distribution of the populations of water molecules with 3 and $4 \mathrm{H}$-bonds show that at $250 \mathrm{~K}$ water molecules with $4 \mathrm{H}$-bonds form an almost percolated network, however, the structure of the population of water molecules with 3 $\mathrm{H}$-bonds is best described as a branched chain network at all temperatures.

Deconvolution of the descriptors of the mixing pattern of water molecules according to their donor and acceptor numbers and show that in general species with complementary H-bonding properties (i.e. with complementary donor and acceptor numbers) are likely to correlate and form hydrogen bonds with each other, while species with similar H-bond pattern tend to avoid each other. Our results also nicely indicate that at low temperatures water molecules with four $\mathrm{H}$-bonds are likely to form $\mathrm{H}$-bonds with each other, supporting the presence of low density patches. Furthermore, the Pearson's coefficient (global descriptor of local cooperativity) of the studied networks suggests that at normal density the H-bonded network in liquid water can be described by an uncorrelated network.

\section{Acknowledgements}

JO acknowledges the financial support of a Bolyai János Research Fellowship and of NKFIH Grant 115503. AL thanks the financial support of Richter Gedeon Talentum. The simulations were performed on resources provided by the Hungarian Supercomputing Facilities (NIIF). IB thanks Uppsala University for hospitality during research visits and 
Hungarian OTKA grant number K108721. I.B thanks for the fruitful discussion with Dénes Berta.

\section{References}

1. Chaplin M. Water structure and science. http://www1.1sbu.ac.uk/water/. Accessed January 8, 2017.

2. Speedy RJ. Limiting forms of the thermodynamic divergences at the conjectured stability limits in superheated and supercooled water. J Phys Chem. 1982;86(15):30023005. doi:10.1021/j100212a038.

3. Poole PH, Sciortino F, Essmann U, Stanley HE. Phase behaviour of metastable water. Nature. 1992;360(6402):324-328. doi:10.1038/360324a0.

4. Sastry S, Debenedetti PG, Sciortino F, Stanley HE. Singularity-free interpretation of the thermodynamics of supercooled water. Phys Rev E. 1996;53(6):6144-6154. doi:10.1103/PhysRevE.53.6144.

5. Stanley HE, Teixeira J, Geiger A, Blumberg RL. Interpretation of the unusual behavior of $\mathrm{H} 2 \mathrm{O}$ and $\mathrm{D} 2 \mathrm{O}$ at low temperature: Are concepts of percolation relevant to the “puzzle of liquid water"? Phys A Stat Mech its Appl. 1981;106(1-2):260-277. doi:10.1016/0378-4371(81)90224-7.

6. Blumberg RL, Stanley HE, Geiger A, Mausbach P. Connectivity of hydrogen bonds in liquid water. J Chem Phys. 1984;80(10):5230-5241. doi:10.1063/1.446593.

7. Oleinikova A, Brovchenko I. Percolating networks and liquid-liquid transitions in supercooled water. J Phys Condens Matter. 2006;18(36):S2247-S2259.

doi:10.1088/0953-8984/18/36/S02.

8. Angell CA. Insights into Phases of Liquid Water from Study of Its Unusual GlassForming Properties. Science (80- ). 2008;319(5863):582-587.

doi:10.1126/science.1131939.

9. Sciortino F, Fornili SL. Hydrogen bond cooperativity in simulated water: Time dependence analysis of pair interactions. J Chem Phys. 1989;90(5):2786-2792. doi:10.1063/1.455927.

10. Kumar P, Franzese G, Buldyrev S V., Stanley HE. Molecular dynamics study of orientational cooperativity in water. Phys Rev E. 2006;73(4):41505.

doi:10.1103/PhysRevE.73.041505.

11. Stanley HE. Understanding Static and Dynamic Heterogeneities in Confined Water. Z Phys Chem. 2009;223:939-956. doi:10.1524.zpch.2009.6064.

12. Holzmann J, Appelhagen A, Ludwig R. Correlation of Static and Dynamic Heterogeneities in Supercooled Water by Means of Molecular Dynamics Simulations. Z Phys Chem. 2009;223:1001-1010. doi:10.1524.zpch.2009.6065.

13. Schmidt DA, Miki K. Structural Correlations in Liquid Water: A New Interpretation of IR Spectroscopy. J Phys Chem A. 2007;111(40):10119-10122. doi:10.1021/JP074737N.

14. Schmidt DA, Miki K. Defective Continuous Hydrogen-Bond Networks: An Alternative Interpretation of IR Spectroscopy. ChemPhysChem. 2008;9(13):1914-1919. doi:10.1002/cphc. 200800236. 
15. Stokely K, Mazza MG, Stanley HE, Franzese G. Effect of hydrogen bond cooperativity on the behavior of water. Proc Natl Acad Sci U S A. 2010;107(4):13011306. doi:10.1073/pnas.0912756107.

16. Pérez C, Zaleski DP, Seifert NA, et al. Hydrogen Bond Cooperativity and the Three-Dimensional Structures of Water Nonamers and Decamers. Angew Chemie Int Ed. 2014;53(52):14368-14372. doi:10.1002/anie.201407447.

17. Frank HS, Wen W-Y. Ion-solvent interaction. Structural aspects of ion-solvent interaction in aqueous solutions: a suggested picture of water structure. Discuss Faraday Soc. 1957;24:133-140. doi:10.1039/df9572400133.

18. Auer B, Kumar R, Schmidt JR, Skinner JL. Hydrogen bonding and Raman, IR, and 2D-IR spectroscopy of dilute HOD in liquid D2O. Proc Natl Acad Sci U S A. 2007;104(36):14215-14220. doi:10.1073/pnas.0701482104.

19. Luzar A. Extent of inter-hydrogen bond correlations in water. Temperature effect. Chem Phys. 2000;258(2):267-276. doi:10.1016/S0301-0104(00)00163-4.

20. Luzar A, Chandler D. Hydrogen-bond kinetics in liquid water. Nature. 1996;379(6560):55-57. doi:10.1038/379055a0.

21. Errington JR, Debenedetti PG. Relationship between structural order and the anomalies of liquid water. Nature. 2001;409(6818):318-321. doi:10.1038/35053024. 22. Errington JR, Debenedetti PG, Torquato S. Cooperative Origin of Low-Density Domains in Liquid Water. Phys Rev Lett. 2002;89(21):215503. doi:10.1103/PhysRevLett.89.215503.

23. Raiteri P, Laio A, Parrinello M. Correlations among Hydrogen Bonds in Liquid Water. Phys Rev Lett. 2004;93(8):87801. doi:10.1103/PhysRevLett.93.087801.

24. Boccaletti S, Latora V, Moreno Y, Chavez M, Hwang D-U. Complex networks: Structure and dynamics. Phys Rep. 2006;424(4):175-308. doi:10.1016/j.physrep.2005.10.009.

25. Dorogovtsev SN, Goltsev A V., Mendes JFF. Critical phenomena in complex networks. Rev Mod Phys. 2008;80(4):1275-1335. doi:10.1103/RevModPhys.80.1275. 26. Albert R, Barabasi A-L. Statistical mechanics of complex networks. Rev Mod Phys. 2002;74:47-97. doi:10.1103/RevModPhys.74.47.

27. Dorogovtsev SN, Mendes JFF. Evolution of networks. Adv Phys. 2002;51(4):1079-1187. doi:10.1080/00018730110112519.

28. Newman MEJ, Strogatz SH, Watts DJ. Random graphs with arbitrary degree distributions and their applications. Phys Rev E. 2001;64(2):26118. doi:10.1103/PhysRevE.64.026118.

29. Onnela J-P, Saramäki J, Hyvönen J, et al. Analysis of a large-scale weighted network of one-to-one human communication. New J Phys. 2007;9(6):179-179. doi:10.1088/1367-2630/9/6/179.

30. Bakó I, Megyes T, Bálint S, et al. Water-methanol mixtures: topology of hydrogen bonded network. Phys Chem Chem Phys. 2008;10(32):5004-5011. doi: $10.1039 / \mathrm{b} 808326 f$.

31. Bakó I, Bencsura Á, Hermannson K, et al. Hydrogen bond network topology in liquid water and methanol: a graph theory approach. Phys Chem Chem Phys. 2013;15(36):15163-15171. doi:10.1039/c3cp52271g.

32. Megyes T, Bálint S, Bakó I, Grósz T, Kótai L, Pálinkás G. Solution structure determination of tetranuclear platinum(II) cluster complex in acetic acid: X-ray 
diffraction and molecular dynamics simulation study. J Mol Liq. 2008;143(1):23-30. doi:10.1016/j.molliq.2008.04.008.

33. Bakó I, Oláh J, Lábas A, Bálint S, Pusztai L, Bellissent Funel MC. Waterformamide mixtures: Topology of the hydrogen-bonded network. J Mol Liq. 2017;228:25-31. doi:10.1016/j.molliq.2016.10.052.

34. Bakó I, Megyes T, Bálint S, et al. Hydrogen bonded network properties in liquid formamide. J Chem Phys. 2010;132(1):14506. doi:10.1063/1.3268626.

35. Lábas A, Bakó I, Oláh J. Hydration sphere structure of proteins: A theoretical study. J Mol Liq. 2017;238:462-469. doi:10.1016/j.molliq.2017.05.038.

36. Wang C-H, Bai P, Siepmann JI, Clark AE. Deconstructing Hydrogen-Bond Networks in Confined Nanoporous Materials: Implications for Alcohol-Water Separation. J Phys Chem C. 2014;118(34):19723-19732. doi:10.1021/jp502867v. 37. Ozkanlar A, Clark AE. ChemNetworks: A complex network analysis tool for chemical systems. J Comput Chem. 2014;35(6):495-505. doi:10.1002/jcc. 23506.

38. Choi J-H, Cho M. Ion aggregation in high salt solutions. II. Spectral graph analysis of water hydrogen-bonding network and ion aggregate structures. J Chem Phys. 2014;141(15):154502. doi:10.1063/1.4897638.

39. Todorov IT, Smith W, Trachenko K, et al. DL_POLY_3: new dimensions in molecular dynamics simulations via massive parallelism. J Mater Chem. 2006;16(20):1911-1918. doi:10.1039/b517931a.

40. Berendsen HJC, Grigera JR, Straatsma TP. The missing term in effective pair potentials. J Phys Chem. 1987;91(24):6269-6271. doi:10.1021/j100308a038.

41. Kumar R, Schmidt JR, Skinner JL. Hydrogen bonding definitions and dynamics in liquid water. J Chem Phys. 2007;126(20):204107. doi:10.1063/1.2742385.

42. Markovitch O, Agmon N. The distribution of acceptor and donor hydrogen-bonds in bulk liquid water. Mol Phys. 2008;106(2-4):485-495.

doi:10.1080/00268970701877921.

43. Campo MG. Structural and dynamic properties of SPC/E water. Pap Phys. 2010;2(0):20001. doi:10.4279/pip.020001.

44. Rick SW. Simulations of ice and liquid water over a range of temperatures using the fluctuating charge model. J Chem Phys. 2001;114(5):2276-2283.

doi:10.1063/1.1336805.

45. Galicia-Andrés E, Dominguez H, Pizio O. Temperature dependence of the microscopic structure and density anomaly of the SPC/E and TIP4P-Ew water models. Molecular dynamics simulation results. Condens Matter Phys. 2015;18:13603. doi:10.5488/CMP.18.13603.

46. Fennell CJ, Li L, Dill KA. Simple Liquid Models with Corrected Dielectric Constants. J Phys Chem B. 2012;116(23):6936-6944. doi:10.1021/jp3002383.

47. Vega C, Sanz E, Abascal JLF. The melting temperature of the most common models of water. J Chem Phys. 2005;122(11):114507. doi:10.1063/1.1862245.

48. Sedlmeier F, Horinek D, Netz RR. Spatial Correlations of Density and Structural Fluctuations in Liquid Water: A Comparative Simulation Study. J Am Chem Soc. 2011;133(5):1391-1398. doi:10.1021/ja1064137.

49. Paton RS, Goodman JM. Hydrogen Bonding and $\pi$-Stacking: How Reliable are Force Fields? A Critical Evaluation of Force Field Descriptions of Nonbonded Interactions. J Chem Inf Model. 2009;49(4):944-955. doi:10.1021/ci900009f. 
50. Ohno K, Okimura M, Akai N, Katsumoto Y, Aoki K, Beu TA. The effect of cooperative hydrogen bonding on the $\mathrm{OH}$ stretching-band shift for water clusters studied by matrix-isolation infrared spectroscopy and density functional theory. Phys Chem Chem Phys. 2005;7(16):3005-3014. doi:10.1039/b506641g.

51. Tao Y, Zou W, Jia J, Li W, Cremer D. Different Ways of Hydrogen Bonding in Water - Why Does Warm Water Freeze Faster than Cold Water? J Chem Theory Comput. 2017;13(1):55-76. doi:10.1021/acs.jctc.6b00735.

52. Baranyai A, Kiss P, Sources of the deficiencies in the popular SPC/E and TIP3P models of water J. Chem. Phys. 2011; 134. 054106 /doi/10.1063/1.3548869 